\title{
Endogenous IL-1 $\alpha$ from systemic sclerosis fibroblasts induces IL-6 and PDGF-A
}

\author{
Yasushi Kawaguchi, ${ }^{1,2}$ Masako Hara, ${ }^{2}$ and Timothy M. Wright ${ }^{1,3}$ \\ ${ }^{1}$ Division of Rheumatology and Clinical Immunology, Department of Medicine, University of Pittsburgh School of Medicine, \\ Pittsburgh, Pennsylvania 15213, USA \\ ${ }^{2}$ Institute of Rheumatology, Tokyo Women's Medical University School of Medicine, Tokyo 162-0054, Japan \\ ${ }^{3}$ Department of Molecular Genetics and Biochemistry and the Pittsburgh Cancer Institute, University of Pittsburgh School of \\ Medicine, Pittsburgh, Pennsylvania 15213, USA \\ Address correspondence to: Yasushi Kawaguchi, Institute of Rheumatology, Tokyo Women's Medical University School of Medicine, \\ 10-22, Kawada-cho, Shinjuku-ku, Tokyo 162-0054, Japan. Phone: 81-3-5269-1725; Fax: 81-3-5269-1726; E-mail: \\ YHK02262@niftyserve.ne.jp
}

Received for publication June 16, 1998, and accepted in revised form March 23, 1999.

It is reported that fibroblasts derived from clinically affected skin areas of patients with systemic sclerosis (SSc) have the ability to overproduce several cytokines and growth factors (i.e., IL-6, PDGF), an ability that might be involved in the pathogenesis of SSc. We have previously shown that the expression of IL-1 $\alpha$ was constitutively observed in SSc fibroblasts, whereas this was not detected in normal fibroblasts. Although it was suggested that the aberrant IL-1 $\alpha$ production could be associated with the fibrogenic phenotype of SSc fibroblasts, little is known about the roles of IL- $1 \alpha$ in SSc fibroblasts. IL- $1 \alpha$ induced IL-6 and PDGF-A, which are potent stimulators of collagen production and proliferation in normal fibroblasts. This article examines the proposal that IL-6 and PDGF-A are elevated through the action of endogenous IL- $1 \alpha$ in SSc fibroblasts. An antisense oligodeoxynucleotide complementary to IL-1 $\alpha$ mRNA was used to suppress endogenous IL-1 $\alpha$. Inhibition of endogenous IL- $1 \alpha$ led to decreased levels of IL-6 and PDGF-A expression in SSc fibroblasts. Moreover, the blocking of the IL-6 response using anti-IL-6 antibody resulted in a significant reduction of procollagen type I in cultured SSc fibroblasts. These results suggest that endogenous IL- $1 \alpha$ expressed by SSc fibroblasts may play a key role in the abnormal function of SSc fibroblasts through the expression of IL- 6 and PDGF-A.

J. Clin. Invest. 103:1253-1260 (1999).

\section{Introduction}

Systemic sclerosis (SSc) is a connective tissue disease characterized by fibrosis not only in skin but also in many internal organs (1). Although the pathogenesis of SSc is still unknown, it is obvious that skin fibrosis results from excessive accumulation of extracellular matrix (2) and that skin fibroblasts from patients with SSc express more collagen mRNA and protein than do normal skin fibroblasts in vivo and in vitro (3-5). A number of researchers have examined the mechanisms of the abnormal functions of SSc fibroblasts (6-10), but many facets of the pathogenesis of SSc remain to be elucidated.

IL- $1 \alpha$ was first described as a factor derived from keratinocytes that stimulates thymocyte proliferation (11). Unlike keratinocytes, other cell types (including dermal fibroblasts) do not constitutively synthesize IL-1 $\alpha$, but they can be stimulated to synthesize and release an active form of IL-1 $\alpha$ (12). The first translational product of IL$1 \alpha$ is pro-IL- $1 \alpha(31 \mathrm{kDa})$, which remains cell associated (13). It is still unclear how IL-1 $\alpha$ is transported from the cytosol to the extracellular compartment and how it is cleaved to its mature peptides $(17.5 \mathrm{kDa})$. Human dermal fibroblasts stimulated by IL- $1 \alpha$ are known to synthesize several kinds of cytokines and growth factors, including IL-1 (14), IL-6 (15), IL-8 (16), TNF- $\alpha$ (17), CSF (18), and PDGF (19). IL-1, IL-6, and PDGF are of prime importance in activating fibroblasts to produce the extracellular matrix.
In an earlier study (20), we demonstrated the aberrant constitutive expression of IL- $1 \alpha$ mRNA in cultured SSc fibroblasts and showed that IL- $1 \alpha$ protein produced by SSc fibroblasts was a 31-kDa precursor form detected only in cell lysates. Because it was reported that pro-IL-1 $\alpha$ was capable of expressing biologic activity through IL- $1 \alpha$ receptor binding on the cell surface (21), we hypothesized that this molecule induces biologic functions in an autocrine manner in SSc-affected fibroblasts. To test this hypothesis, the present study investigates the inhibitory effect of antisense oligodeoxynucleotides (ODNs) complementary to IL- $1 \alpha$ mRNA on the expression of IL1 -inducible protein and genes by cultured SSc fibroblasts.

\section{Methods}

Cell cultures. Human skin fibroblasts from 9 clinically affected and 3 clinically unaffected areas of 9 patients with SSc, all of whom conformed to the American College of Rheumatology criteria (22) for SSc, and from 5 healthy donors, were cultured as described previously (23). All experiments were performed with 3- to 5-passage cultures.

Antisense ODN to buman IL-1 $\alpha m R N A$. To evaluate the role of endogenous IL- $1 \alpha$ in SSc fibroblasts, an antisense ODN complementary to IL- $1 \alpha$ mRNA was used to suppress endogenous IL-1 $\alpha$ protein expression. A phosphorothioate analogue of antisense ODN to IL- $1 \alpha$ mRNA, which was designed to bind 9 nucleotides upstream and downstream from the translation initiation codon (ATG), was synthesized by Bio-Synthesis Inc. (Lewisville, Texas, USA). The sequence of antisense ODN was 
Table 1

mRNA levels of IL-1 $\alpha$ in SSc-affected fibroblasts treated with antisense ODN

Levels of transcripts (copy number/ $\mu \mathrm{g}$ total RNA)

Control + Antisense ODN $\left(\right.$ ratio $\left.^{A}\right)+$ Sense ODN $\left(\right.$ ratio $\left.^{A}\right)$

Affected fibroblasts

$\begin{array}{lll}1.5 \times 10^{4} & 0.2 \times 10^{4}(0.132) & 1.9 \times 10^{4}(1.304) \\ 2.1 \times 10^{4} & 0.3 \times 10^{4}(0.145) & 1.4 \times 10^{4}(0.667) \\ 1.3 \times 10^{3} & 0.5 \times 10^{3}(0.385) & 2.1 \times 10^{3}(1.615) \\ 1.0 \times 10^{4} & 1.8 \times 10^{3}(0.180) & 8.9 \times 10^{3}(0.890) \\ 5.5 \times 10^{3} & 1.0 \times 10^{3}(0.181) & 5 \times 10^{3}(0.909)\end{array}$

Mean $\pm \mathrm{SD} \times 10^{-3}$

$11 \pm 7.8 \quad 1.7 \pm 1.0^{\mathrm{B}}$

$9.8+6.8$

Five affected SSc fibroblasts were stimulated with sense or antisense ODN $(20 \mu \mathrm{M})$ for $48 \mathrm{~h}$, and then total RNA was extracted. Competitive RT-PCR analysis for IL- $1 \alpha$ mRNA was performed, and values of transcripts were calculated by the graph as described in Methods. ${ }^{A} \mathrm{Ra}$ tio indicates copy number of ODN-treated cells/control cells. ${ }^{\mathrm{B}} P<0.05$ compared with control by paired Student's $t$ test.

5'-TTT GGC CAT CTT GAC TTC-3', and the sequence of sense ODN, used as a control, was 5'-GAA GTC AAG ATG GCC AAA$3^{\prime}$, as described previously (24).

Measurement of IL-1 $\alpha$ in antisense ODN-treated fibroblasts. Confluent fibroblasts were cultured with serum-free medium (QBSF-51; Sigma Chemical Co., St. Louis, Missouri, USA) containing various concentrations of sense or antisense ODN. The following day, cells were harvested and cell lysates were collected at $-20^{\circ} \mathrm{C}$. The amount of IL- $1 \alpha$ was measured using an ELISA kit (R\&D Systems Inc., Minneapolis, Minnesota, USA).

Measurement of IL- 6 in antisense ODN-treated fibroblasts. Monolayer confluent fibroblasts were cultured with serum-free medium containing $20 \mu \mathrm{M}$ of sense or antisense ODN for 48 hours. The medium was exchanged for fresh serum-free medium with $20 \mu \mathrm{M}$ of sense or antisense ODN. After further culturing for another 24 hours, the amounts of IL- 6 in the supernatants were assayed using an ELISA kit (Genzyme Pharmaceuticals, Cambridge, Massachusetts, USA).

$R N A$ isolation and quantitative RT-PCR. Fibroblasts treated with various concentrations of sense or antisense ODN were cultured in serum-free medium for the indicated time. Total RNA was extracted from fibroblast cultures using Trizol (GIBCO BRL, Grand Island, New York, USA). RT-PCR was performed using an RNA PCR kit (Perkin-Elmer Cetus, Norwalk, Connecticut, USA). Briefly, $1 \mu \mathrm{g}$ of total RNA from each sample was reverse transcribed to cDNA together with various concentrations $\left(10^{3}-10^{6}\right.$ copies) of pAW109 (Perkin-Elmer Cetus) or pGEMIL-6 (made as follows and used as an internal RNA control). The RT reaction was performed at $42^{\circ} \mathrm{C}$ for 50 minutes in a volume of $20 \mu \mathrm{L} ; 2 \mu \mathrm{L}$ of each RT solution was then used for PCR in a volume of $50 \mu \mathrm{L}$. Thirty cycles of PCR were performed with a set of primers for IL$1 \alpha$ and PDGF-A mRNA described by Wang et al. (25), and with a set of primers for IL-6 (sense: $5^{\prime}$-ATG AAC TCC TTC TCC ACA AGC GCC TTC-3'; antisense: 5'-TGG CTT GTT CCT CAC TAC TCT CAA ATC-3'; product size: 421 bp). The PCR samples were electrophoresed in $2 \%$ agarose gels (to confirm the size of PCR products) and then in $8 \%$ acrylamide gels. The acrylamide gels were vacuum dried, and the radioactive signals on the gel were analyzed using an image acquisition and analysis system (PhosphorImager SI; Molecular Dynamics Inc., Sunnyvale, California, USA). The ratio of the signal obtained from the internal control and the target cellular RNA was plotted against a copy number of the internal control used. The copy numbers of target mRNA were calculated from the graph by correcting for the number of $\mathrm{dCTP}$ residues contained in each PCR product.
Synthesis of internal RNA control for IL-6. A partial IL-6 cDNA (421 bp) was made by PCR using the primers already indicated here. The cDNA was inserted into pGEM-T vector (Promega Corp., Madison, Wisconsin, USA), which was then cut by EcoNI (New England Biolabs Inc., Beverly, Massachusetts, USA) at a unique restriction site located at the center of IL- 6 cDNA. A 120 -bp DNA fragment made by PCR was inserted into the digested PGEM-T vector using T4 DNA ligase (New England Biolabs Inc.). In vitro transcription was performed from the SP6 promotor site using a MAXIscript kit (Ambion Inc., Austin, Texas, USA). The RNA obtained was used for the IL- 6 internal control and referred to as PGEMIL-6.

Measurement of procollagen type I produced by fibroblasts. Fibroblasts $\left(5 \times 10^{4}\right.$ cells) were cultured in 24 -well culture plates with DMEM plus 10\% FBS, complemented by various concentrations of mouse monoclonal anti-human IL-6 antibody (Genzyme Pharmaceuticals). Ten wells were used for each experiment, starting at day 0 . The following day (day 1 ), supernatants of 2 wells were collected and stored at $-80^{\circ} \mathrm{C}$, cells were trypsinized, and cell numbers were counted. On days 3 and 5 , supernatants of 2 more wells were collected and cell numbers were counted. On day 5 , the media of 4 wells were aspirated; cells were washed with PBS 3 times, and new media (including various concentrations of anti-IL-6 antibody) were added. On days 7 and 10, supernatants of 2 wells were collected and cell numbers were counted. Procollagen type I production of each culture supernatant was estimated by a procollagen type I c-peptide ELISA kit (Takara Shuzo, Otsu, Japan).

Immunohistochemistry. Frozen sections of skin biopsies in OCT compound from 3 patients with SSc and 2 healthy controls were investigated for IL-1 $\alpha$ using rabbit polyclonal anti-human IL$1 \alpha$ antibody $(5 \mu \mathrm{g} / \mathrm{mL}$; Genzyme Pharmaceuticals). Frozen sections $(5 \mu \mathrm{m})$ were fixed by air drying and were incubated with anti-human IL- $1 \alpha$ antibody or preimmune rabbit IgG at room temperature for 1 hour. All sections were washed 3 times for 5

\section{Table 2}

mRNA levels of IL-6 in SSc-affected and -unaffected fibroblasts treated with antisense ODN

Levels of transcripts (copy number/ $\mu$ g total RNA) Control + Antisense ODN $\left(\right.$ ratio $\left.^{A}\right)$ +Sense ODN $\left(\right.$ ratio $\left.^{A}\right)$

Affected fibroblasts

$\begin{array}{ll}6.2 \times 10^{6} & 5.5 \times 10^{5}(0.089) \\ 3.8 \times 10^{6} & 7.0 \times 10^{5}(0.184) \\ 5.3 \times 10^{6} & 8.2 \times 10^{5}(0.155) \\ 1.9 \times 10^{6} & 2.8 \times 10^{5}(0.147) \\ 1.7 \times 10^{6} & 2.9 \times 10^{5}(0.171)\end{array}$
$4.5 \times 10^{6}(0.726)$ $4.7 \times 10^{6}(1.237)$ $4.7 \times 10^{6}(0.887)$ $1.6 \times 10^{6}(0.842)$ $2.1 \times 10^{6}(1.235)$

Mean $\pm \mathrm{SD} \times 10^{-5}$

$$
38 \pm 20 \quad 5.3 \pm 2.4^{\mathrm{B}} \quad 35 \pm 15
$$

Unaffected fibroblasts

$$
\begin{array}{ccc}
3 \times 10^{5} & 2.7 \times 10^{5}(0.9) & 3.3 \times 10^{5}(1.1) \\
3.1 \times 10^{5} & 2.6 \times 10^{5}(0.839) & 2.7 \times 10^{5}(0.871)
\end{array}
$$

Mean $\pm \mathrm{SD} \times 10^{-5}$

$$
3.1 \pm 0.1
$$

$$
2.7 \pm 0.1
$$

$3.0 \pm 0.4$

Five affected and two unaffected SSc fibroblasts were stimulated with sense or antisense ODN $(20 \mu \mathrm{M})$ for $48 \mathrm{~h}$, and then total RNA was extracted. Competitive RTPCR analysis for IL-6 mRNA was performed, and values of transcripts were calculated by the graph as described in Methods.

ARatio indicates copy number of ODN-treated cells/control cells. ${ }^{B} P<0.05$ compared with control by paired Student's $t$ test. 
minutes with PBS, followed by incubation with biotinylated horse anti-rabbit IgG (Vector Laboratories, Burlingame, California, USA) for 30 minutes. The sections were incubated with avidin-biotin complex (ABC; Vector Laboratories) and developed with 3,3' diaminobenzidine tetrahydrochloride (DAB) and $\mathrm{H}_{2} \mathrm{O}_{2}$ for 10 minutes.

Statistical analysis. Results are expressed as mean \pm SD. Statistical comparisons were performed using Student's $t$ test for paired and unpaired data. $P$ values $<0.05$ were considered significant for all tests.

\section{Results}

Effect of antisense ODN against IL-1 $\alpha$ mRNA on the expression of $I L-1 \alpha$. To study whether IL- $1 \alpha$ is constitutively produced in SSc-affected fibroblasts, IL- $1 \alpha$ production was analyzed by ELISA in SSc-affected and -unaffected fibroblasts and in normal fibroblasts. As shown in Figure 1a, IL$1 \alpha$ was constitutively produced in SSc-affected fibroblasts $\left(67.8 \pm 35 \mathrm{pg} / 10^{5}\right.$ cells) but was not detected in SSc-unaffected fibroblasts or normal fibroblasts. To evaluate the effect of endogenous IL- $1 \alpha$ on the phenotype of SScaffected fibroblasts, we suppressed IL- $1 \alpha$ production using antisense ODN complementary to IL- $1 \alpha$ mRNA. Fibroblasts from 9 patients with SSc were cultured with various concentrations $(10-50 \mu \mathrm{M})$ of antisense ODN for 48 hours; IL-1 $\alpha$ production was significantly decreased at concentrations exceeding $20 \mu \mathrm{M}$ of antisense ODN (Figure 1b). Also, fibroblasts from 6 patients with SSc were cultured with $20 \mu \mathrm{M}$ of antisense ODN for up to 72 hours; IL-1 $\alpha$ production was significantly decreased after 48 hours' exposure to antisense ODN (Figure 1c).

A competitive RT-PCR method was carried out to examine the mRNA expression levels of IL- $1 \alpha$ genes by SScaffected fibroblasts. Fibroblasts from 5 patients with SSc were cultured with $20 \mu \mathrm{M}$ of antisense ODN for 48 hours, and total RNA was extracted. Both total RNA from fibroblasts and various copy numbers of control RNA were reverse transcribed in a $0.5-\mathrm{mL}$ tube, and then the $\mathrm{PCR}$ reaction was carried out using the cDNA. Representative results are shown in Figure 2. The steady-state levels of IL- $1 \alpha$ mRNA were decreased by $20 \mu \mathrm{M}$ of antisense ODN, and the differences were significant by paired Student's $t$ test (Table 1).

Effect of antisense ODN against IL-1 $\alpha m R N A$ on the expression of IL-6. To determine whether aberrant IL- $1 \alpha$ production could be involved in overproduction of IL-6 in SSc fibroblasts, we investigated the effect of antisense ODN complementary to IL- $1 \alpha$ mRNA on IL- 6 production. Fibroblasts from affected and unaffected skin areas in 9 patients with SSc were cultured with 10 or $20 \mu \mathrm{M}$ of antisense or sense ODN. As shown in Figure 3, IL-6 production was decreased by the antisense ODN in SSc fibroblasts from affected areas $(n=9)$, but not in SScunaffected fibroblasts $(n=3)$ or in fibroblasts from normal donors $(n=5)$. To examine the effect of the inhibition of IL- $1 \alpha$ on IL- 6 mRNA expression, a quantitative RT-PCR for IL- 6 mRNA was performed on fibroblasts from 5 affected and 3 unaffected areas in patients with SSc. A representative example is shown in Figure 4, and a summary is shown in Table 2 . The steady-state levels of IL-6 mRNA were significantly decreased in SSc-affected fibroblasts by $20 \mu \mathrm{M}$ of antisense $\mathrm{ODN}(P<0.05)$, whereas there was no significant change in SSc-unaffected a

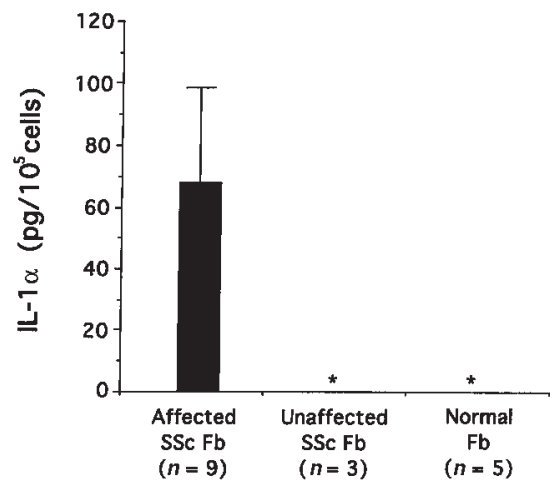

b

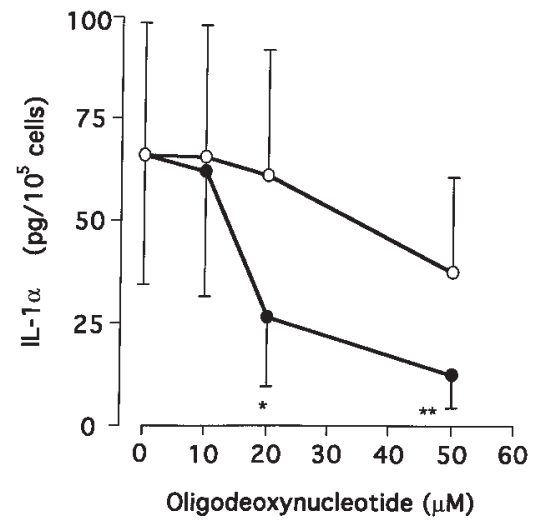

C

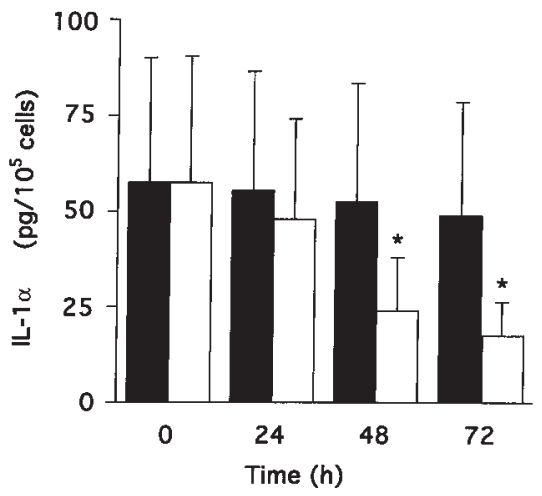

Figure 1

(a) Production of IL- $1 \alpha$ in affected and unaffected fibroblasts from patients with SSc and in normal fibroblasts. Fibroblasts were cultured in serum-free media for 24 hours, and cell lysates were collected. IL- $1 \alpha$ concentrations in cell lysates were measured by an ELISA kit. The results are expressed as the mean \pm SD. Asterisks indicate IL- $1 \alpha$ concentrations below the sensitivity of the ELISA kit used. (b) Effect of antisense ODN complementary to IL- $1 \alpha$ mRNA on IL- $1 \alpha$ production in SSc-affected fibroblasts $(n=9)$. SSc-affected fibroblasts were cultured in serum-free medium with various concentrations of sense (open circles) or antisense (filled circles) ODN for 48 hours. Cell lysates were collected, and IL- $1 \alpha$ production was measured using an ELISA kit. The results are expressed as the mean \pm SD of IL- $1 \alpha$ production. ${ }^{*} P<0.05,{ }^{*} P<0.01$ compared with sense ODN (control) by paired Student's $t$ test. (c) Time course of $\mathrm{IL}-1 \alpha$ production in SSc-affected fibroblasts treated with IL- $1 \alpha$ antisense ODN. SSc-affected fibroblasts $(n=6)$ were cultured in serum-free medium with $20 \mu \mathrm{M}$ of sense (filled bars) or antisense (open bars) ODN for the indicated time. Cell lysates were extracted, and IL- $1 \alpha$ production was measured using an ELISA kit. The results are expressed as the mean \pm SD of IL- $1 \alpha$ production. ${ }^{*} P<0.05$ compared with sense ODN (control) by paired Student's $t$ test. 
Table 3

mRNA levels of PDGF-A in SSc-affected and -unaffected fibroblasts treated with antisense ODN

Levels of transcripts (copy number/ $\mu \mathrm{g}$ total RNA)

Control + Antisense $\operatorname{OD}\left(\right.$ ratio $\left.^{A}\right)+$ Sense $\operatorname{ODN}\left(\right.$ ratio $\left.^{A}\right)$

Affected fibroblasts

$\begin{array}{ll}1.1 \times 10^{6} & 3.8 \times 10^{4}(0.035) \\ 7.6 \times 10^{5} & 3.4 \times 10^{4}(0.045) \\ 4.1 \times 10^{5} & 2.3 \times 10^{4}(0.055) \\ 1.2 \times 10^{6} & 4.0 \times 10^{5}(0.333) \\ 7.1 \times 10^{5} & 5.8 \times 10^{3}(0.008)\end{array}$

$10^{6}(0.909)$

$6 \times 10^{5}(0.789)$

$4.8 \times 10^{5}(1.171)$

$1.2 \times 10^{6}(1.000)$

$5.4 \times 10^{5}(0.761)$

Mean $\pm \mathrm{SD} \times 10^{-5}$

$$
8.4 \pm 3.2 \quad 1.1 \pm 1.6^{\mathrm{C}} \quad 7.6 \pm 3.2
$$

Unaffected fibroblasts

$$
\begin{array}{ll}
1.1 \times 10^{5} & 1.2 \times 10^{5}(1.091) \\
1.1 \times 10^{5} & 1.6 \times 10^{5}(1.454)
\end{array}
$$

$1.3 \times 10^{5}(1.182)$

$9.6 \times 10^{4}(0.872)$

Mean $\pm \mathrm{SD} \times 10^{-5}$

$$
\begin{array}{lll}
1.1 \pm 0.0^{\mathrm{B}} & 1.4 \pm 0.3 & 1.1 \pm 0.2
\end{array}
$$

Five affected and two unaffected SSc fibroblasts were stimulated with sense or antisense ODN $(20 \mu \mathrm{M})$ for $48 \mathrm{~h}$, and then total RNA was extracted. Competitive RTPCR analysis for PDGF-A mRNA was performed, and values of transcripts were calculated by the graph as described in Methods. ARatio indicates copy number of ODN-treated cells/control cells. ${ }^{B} P<0.05$ compared with affected fibroblasts by unpaired Student's $t$ test. ${ }^{C} P<0.01$ compared with control by paired Student's $t$ test.

fibroblasts. We confirmed that this quantitative RT-PCR method was reproducible when duplicate determinations were performed on separate days.

Effect of anti-human IL-6 antibody on procollagen type I production. To determine whether overexpression of IL-6 might be involved in excessive collagen production, we inhibited IL-6 activity using anti-human IL-6 antibody. Given that the old media were discarded and fresh media (including various concentrations of anti-IL-6 antibody) were added to each well on day 5 (as described in Methods), the values of procollagen production on days 7 and 10 represent the net values of procollagen production on days 7 and 10 plus the values on day 5 . Procollagen production was significantly decreased in SSc-affected fibroblasts $(n=$ 2) treated with anti-IL-6 antibody for more than 7 days, compared with SSc-affected fibroblasts with mouse IgG as a control (Figure 5, a and b). In contrast, there was no detectable effect of anti-IL-6 antibody on procollagen production in SSc-unaffected fibroblasts $(n=2)$.

Effect of antisense ODN against IL-1 $\alpha$ mRNA on PDGF-A $m R N A$ expression. Because PDGF protein is a mitogenic factor of fibroblasts and is able to be induced by IL- $1 \alpha$, we examined the effect of antisense ODN against IL- $1 \alpha$ mRNA on PDGF-A mRNA expression of SSc-affected fibroblasts. The expression of PDGF-A mRNA was estimated using a quantitative RT-PCR method. These experiments were performed on fibroblasts from 5 affected and 2 unaffected areas of 5 patients with SSc. A representative example of the results is shown in Figure 6 , and a summary is presented in Table 3 . The results indicate that the levels of PDGF-A mRNA are decreased in SSc-affected fibroblasts by the reduction of endogenous IL- $1 \alpha$ production, whereas there was no significant change in SSc-unaffected fibroblasts. The steady-state levels of PDGF-A mRNA in 5 SSc-affected fibroblasts were higher than the levels in 2 SSc-unaffected fibroblasts, and the difference was significant with unpaired Student's $t$ test. We confirmed that this RT-PCR method was reproducible when duplicate determinations were performed on separate days.

Expression of IL-1 $\alpha$ in SSc skin. This study in vitro revealed that endogenous IL- $1 \alpha$ could induce IL- 6 and PDGF-A expression, which might be involved in the phenotype of $\mathrm{SSc}$ fibroblasts, but it remains to be fully confirmed in vivo. To investigate whether IL-1 $\alpha$ is present in SSc lesional skin, immunostaining for IL- $1 \alpha$ of SSc and control skins was performed. Representative results were shown in Figure 7 of the staining observed in 2 SSc skin biopsies (Figure 7, c and d) compared with 2 normal controls (Figure 7, a and b). We observed IL- $1 \alpha$-positive fibroblasts in skin tissues from 3 patients with SSc, whereas control skin biopsies were negative for IL- $1 \alpha$ staining (Table 4).

\section{Discussion}

Our earlier study (20) indicated that SSc-affected fibroblasts constitutively express cell-associated IL- $1 \alpha$ that is absent in normal fibroblasts, but the function of endogenous IL-1 $\alpha$ in SSc-affected fibroblasts was not elucidated. In the present study, we demonstrate that the aberrant production of IL- $1 \alpha$ contributes to abnormal regulation of both IL- 6 and PDGF-A expression, which may in turn result in excessive collagen synthesis in SScaffected fibroblasts.

The use of antisense ODN can be a useful technique to inhibit the synthesis of proteins (26). The size and target sequences are important considerations in the design of antisense ODN. In general, antisense ODNs directed to 5 ' regions of eukaryotic mRNA, particularly near the initiation codon sequences, are more effective than those directed to $3^{\prime}$ regions $(27,28)$. Therefore, an antisense ODN complementary to the initiation codon sequences of the IL- $1 \alpha$ gene was used in our study. The inhibitory

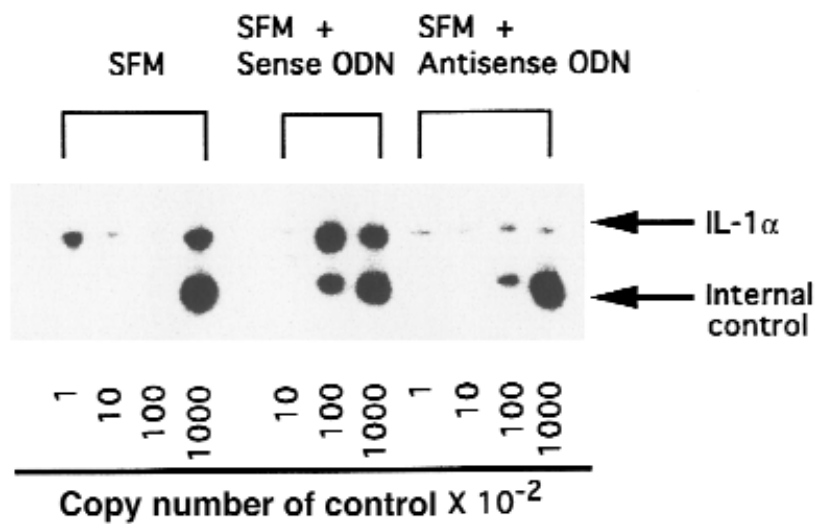

Figure 2

Quantitative RT-PCR of IL- $1 \alpha$ mRNA derived from fibroblasts in patients with SSc. A 1- $\mu$ g aliquot of the total RNA from fibroblasts was analyzed with the internal control ( $P A W 109$ ) containing the indicated number of control RNA copies using RT-PCR. PCR products were electrophoresed using $8 \%$ polyacrylamide gels. The polyacrylamide gels were dried and exposed to $\mathrm{x}$-ray film with intensifier screens for 18 hours at $-80^{\circ} \mathrm{C}$. 


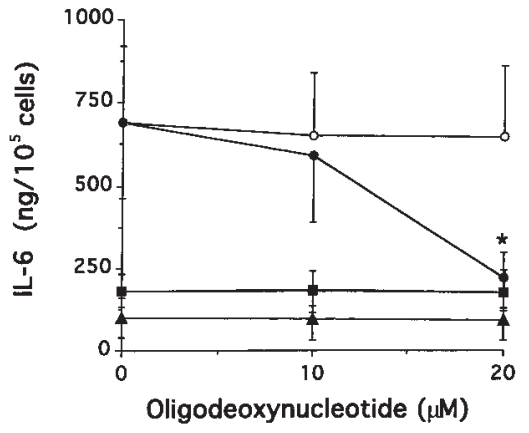

\section{Figure 3}

Effect of the inhibition of endogenous IL- $1 \alpha$ on IL- 6 production in SSCaffected fibroblasts. Fibroblasts from affected skin areas of 9 patients with SSc (circles), unaffected skin areas of 3 patients with SSc (squares), and 5 normal donors (triangles) were cultured in serum-free medium in the presence of IL-1 $\alpha$ sense or antisense ODN $(20 \mu \mathrm{M})$ for 48 hours. The medium was exchanged for fresh serum-free medium with $20 \mu \mathrm{M}$ of sense (open symbols) or antisense (filled symbols) ODN. After further culturing for another 24 hours, the amount of IL- 6 in the culture supernatant was measured using an ELISA kit. The results are indicated as mean \pm SD. ${ }^{*} P<0.001$ compared with cells treated with sense ODN by paired Student's $t$ test.

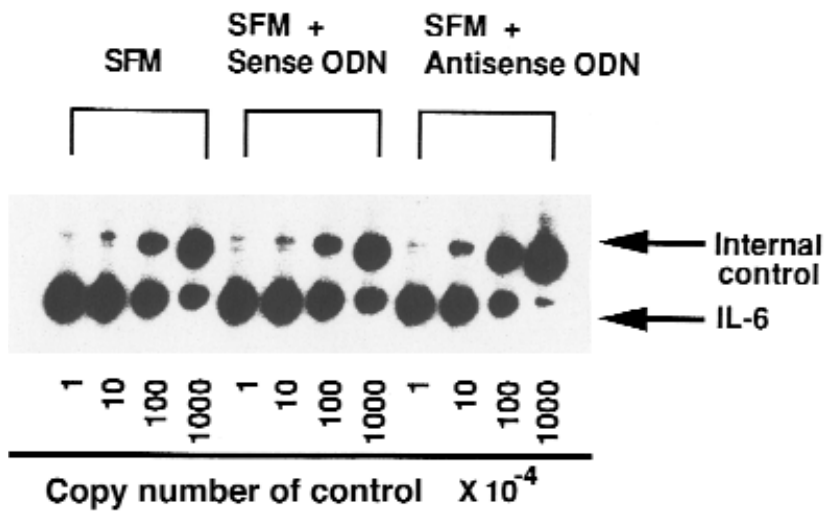

Figure 4

Representative results of quantitative RT-PCR of IL- 6 mRNA derived from $\mathrm{SSc}$-affected fibroblasts. A 1- $\mu \mathrm{g}$ aliquot of the total RNA from affected and unaffected fibroblasts in patients with SSc was analyzed with the internal control (pGEMIL-6) containing the indicated number of control RNA copies using RT-PCR. PCR products were electrophoresed using $8 \%$ polyacrylamide gels. The polyacrylamide gels were dried and exposed to $x$-ray film with intensifier screens for 18 hours at $-80^{\circ} \mathrm{C}$.

effects of antisense ODN may be attributable to the degradation of mRNA by RNase activity in cells (29) or to translational arrest (30). Because our studies showed the inhibitory effect of antisense ODN on mRNA and protein levels of IL-1 $\alpha$, the antisense ODN used in the present study could mediate the degradation of IL- $1 \alpha$ mRNA.

Human dermal fibroblasts stimulated by IL- $1 \alpha$ are known to produce IL-6 (15), and several researchers have shown that the production of IL-6 is increased spontaneously in SSc-affected fibroblasts compared with that in SSc-unaffected fibroblasts and normal fibroblasts in vitro $(10,31,32)$. The levels of serum IL-6 have been reported to be higher in patients with SSc than in normal, healthy controls (33). Also, increased IL-6 levels in bronchoalveolar lavage fluid have been found in patients with SSc (34). These findings suggest that IL-6 might play a role in the pathogenesis of fibrosis in SSc. This evidence prompted us to determine whether or not an overproduction of IL-6 is a consequence of constitutive IL- $1 \alpha$ production. To evaluate the role of IL- $1 \alpha$, we decreased the endogenous IL- $1 \alpha$ of SSc-affected fibroblasts using antisense ODN against IL- $1 \alpha$ mRNA. Antisense ODN has been used to block the transcription of genes and/or the translation of proteins in cultured cells (35), but the use of natural ODN in studies of normal cells is limited by the rapid metabolismof ODN by endogenous nucleases. Therefore, we used the phosphorothioate analogue of ODN, which is more nuclease resistant (36). After the suppression of endogenous IL-1 $\alpha$ protein production, the values of IL6 showed an expected significant reduction in SScaffected fibroblasts. On the other hand, the value of IL6 was not changed in the cases of SSc-unaffected and normal fibroblasts treated with antisense ODN. These results indicated that overproduction of IL- 6 depends in part on the action of endogenous IL- $1 \alpha$.

Because it is clear that skin fibrosis in patients with SSc results from excessive collagen production by skin fibroblasts and that IL- $1 \alpha$ and IL- 6 are potent inducers of collagen production $(37,38)$, we examined the effects of antisense ODN against IL- $1 \alpha$ mRNA and anti-IL-6 antibody on procollagen production. Although we initially examined the effect of antisense ODN $(20 \mu \mathrm{M})$ on procollagen type I production by SSc-affected fibroblasts, the inhibitory effect was not significant (data not shown). We speculate that the effect of ODN might be too transient and too weak to affect collagen production through a decrease in IL-1 $\alpha$ protein or that the effect of IL-1 $\alpha$ on collagen synthesis might be mediated by other proteins. Our next strategy involved the administration of mouse monoclonal antibody against human IL-6. The antibody significantly inhibited procollagen production in SScaffected fibroblasts. This observation revealed that increased synthesis of collagen in SSc-affected fibroblasts was implicated with the aberrant production of IL-6, which could be mediated by the autocrine effect of IL- $1 \alpha$. However, procollagen production did not decrease to the level of procollagen produced by fibroblasts from unaffected areas of patients with SSc. Additional experiments using higher concentrations of anti-IL-6 antibody $(50 \mu \mathrm{g} / \mathrm{mL}$ and $100 \mu \mathrm{g} / \mathrm{mL})$ were performed, and the results did not demonstrate any

\section{Table 4}

Proportion of IL-1 $\alpha$-positive fibroblasts in skin

$\begin{array}{lc} & \text { Number of IL-1 } \alpha \text {-positive fibroblasts } \\ \text { SSc- } 1 & 18 \\ \text { SSc- } 2 & 11 \\ \text { SSc-3 } & 27 \\ & \\ \text { NC-1 } & \text { ND } \\ \text { NC-2 } & \text { ND }\end{array}$

Frozen sections of skin from systemic sclerosis (SSc) and normal controls (NC) were studied using anti-IL- $1 \alpha$ antibody. The numbers of IL- $1 \alpha$-positive cells were counted in 3 different microscopic fields $(\times 200)$. ND, not detectable. 
a

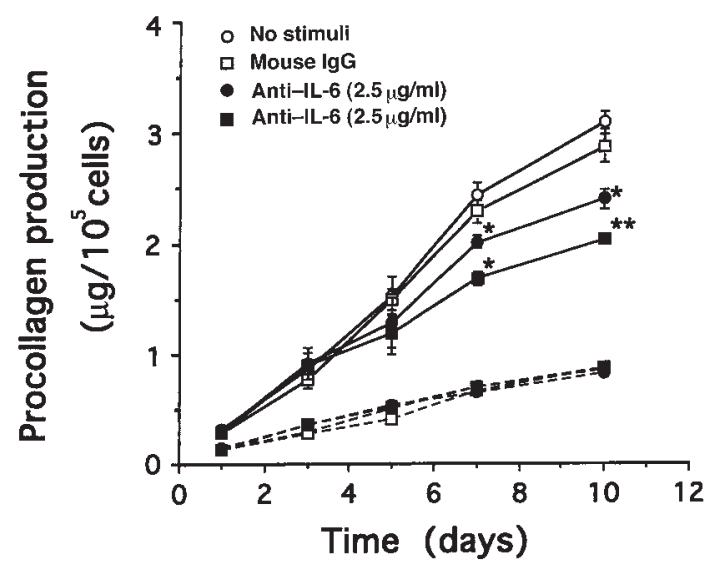

b

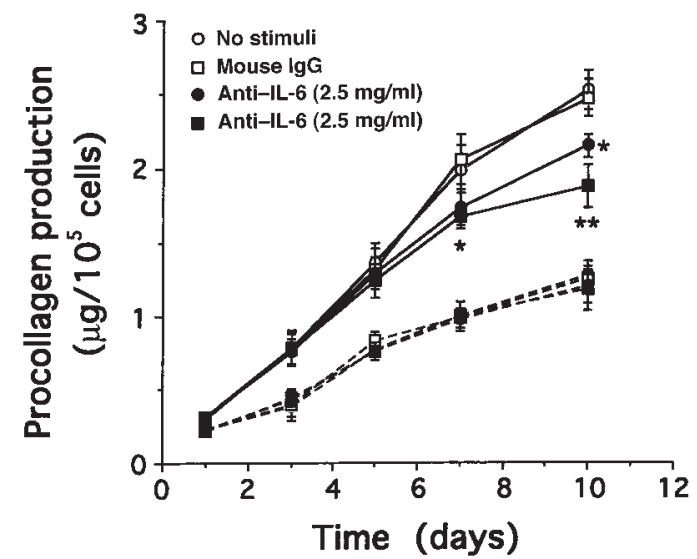

Figure 5

Effect of anti-IL-6 antibody on procollagen production in affected and unaffected fibroblasts derived from 2 patients with SSc. Fibroblasts were cultured with DMEM plus 10\% FBS in the presence of anti-IL-6 antibody ( 2.5 or $25 \mu \mathrm{g} / \mathrm{mL}$ ) in 24-well culture dishes for the indicated number of days. In the culture supernatants, procollagen production was measured by an ELISA kit. The solid and dashed lines indicate the results of SScaffected and -unaffected fibroblasts, respectively. The results are expressed as mean \pm SD of triplicate determinations. (a) SSc patient 1 : ${ }^{*} P<0.05$, ${ }^{*} P<0.01$ compared with fibroblasts with mouse $\lg G$. (b) SSc patient 2 : ${ }^{*} P<0.05,{ }^{*} P<0.01$ compared with fibroblasts with mouse IgG.

dose-dependent inhibition of procollagen production by SSc-affected fibroblasts (data not shown). These results suggest that neutralizing IL-6 activity is not sufficient to attenuate collagen synthesis of SSc fibroblasts to normal levels. There are 2 possibilities that may explain these observations. First, several reports have demonstrated the involvements of TGF$\beta$ (39), PDGF (8), and IL-4 (40) in SSc fibrosis. It is possible that multiple factors including IL- 6 and IL- $1 \alpha$ may be associated with the mechanisms of SSc fibrosis. Second, Hasegawa et al. (33) found that the levels of soluble IL-6 receptor were elevated in sera from patients with SSc, and they speculated that the main sites of IL- 6 receptor expression in vivo might be the liver and skin. This evidence suggests that the response of IL- 6 may be upregulated in SSc-affected fibroblasts because of an increase in IL- 6 receptors expressed in fibroblasts. One possibility is that it may be difficult to block the IL- 6 action completely using high concentrations of anti-IL- 6 antibody because of the aug- mentation of IL- 6 response in SSc-affected fibroblasts. IL-1 $\alpha$ was shown to modulate the mitogenic response of normal fibroblasts, and the mitogenic action was indirect and mediated by the induction of PDGF-A synthesis (19). Using immunohistochemistry methods, Yamakage et al. (8) found that PDGF-A was present in the skin of patients with SSc, whereas it was essentially absent in healthy skin. Although they did not show the source of inappropriate expression of PDGF-A, the local aberrant production of PDGF-A might contribute to the expansion of connective tissue cells and collagen accumulation in the skin of patients with SSc because PDGF-A is a potent chemoattractant and mitogen for fibroblasts and a stimulator of collagen synthesis by fibroblasts. In the present study, it has been shown that SSc-affected fibroblasts exhibit higher expression of PDGF-A mRNA in contrast to SSc-unaffected fibroblasts. Therefore, we examined the effect of antisense ODN on the mRNA level of PDGF-A in cultured SSc fibroblasts and found that the expression of PDGF-A mRNA in SSc fibroblasts decreased with the treatment of antisense ODN to IL- $1 \alpha$ mRNA. Because the inhibitory effect was not observed in SSc-unaffected fibroblasts, which did not produce IL-1 $\alpha$ constitutively, it is strongly suggested that an increased expression of PDGF-A mRNA in SSc-affected fibroblasts is downstream of the aberrant constitutive IL- $1 \alpha$ production in these cells. We speculate that PDGF-A and IL- 6 may be involved in the pathogenesis of the fibrosis in SSc.

In the present study, we emphasized that endogenous IL- $1 \alpha$ could play a crucial role in the phenotype of SScaffected fibroblasts in vitro. Also, we found that IL- $1 \alpha$ is consistently observed in fibroblast-like cells of SSc dermis, whereas it is essentially absent in healthy skin. Characteristics of SSc fibroblasts such as increased production of IL- $1 \alpha$, IL-6, and PDGF-A probably contribute to excessive extracellular matrix synthesis and abnormal proliferation leading to tissue fibrosis.

In conclusion, it is clear that constitutive endogenous IL-

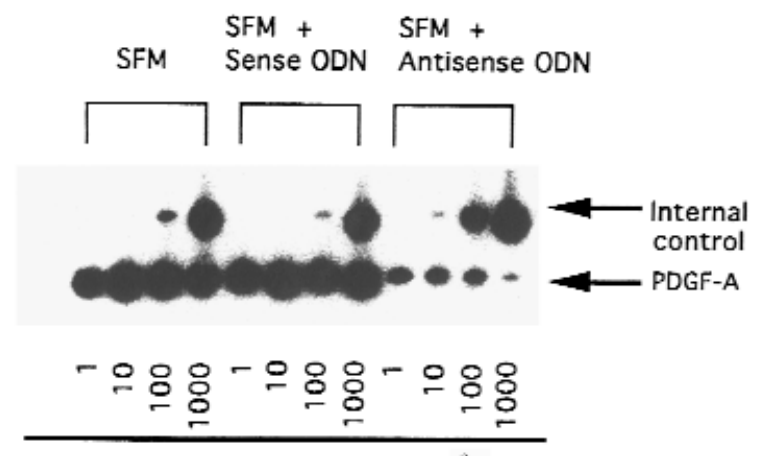

Copy number of control $\times 10$

\section{Figure 6}

Representative results of quantitative RT-PCR of PDGF-A mRNA derived from SSc-affected fibroblasts. A 1- $\mu$ g aliquot of the total RNA from affected and unaffected fibroblasts in patients with SSc was analyzed, with the internal control (pAW109) containing the indicated number of control RNA copies using RT-PCR. PCR products were electrophoresed using $8 \%$ polyacrylamide gels. The polyacrylamide gels were dried and exposed to $\mathrm{x}$-ray film with intensifier screens for 18 hours at $-80^{\circ} \mathrm{C}$. 

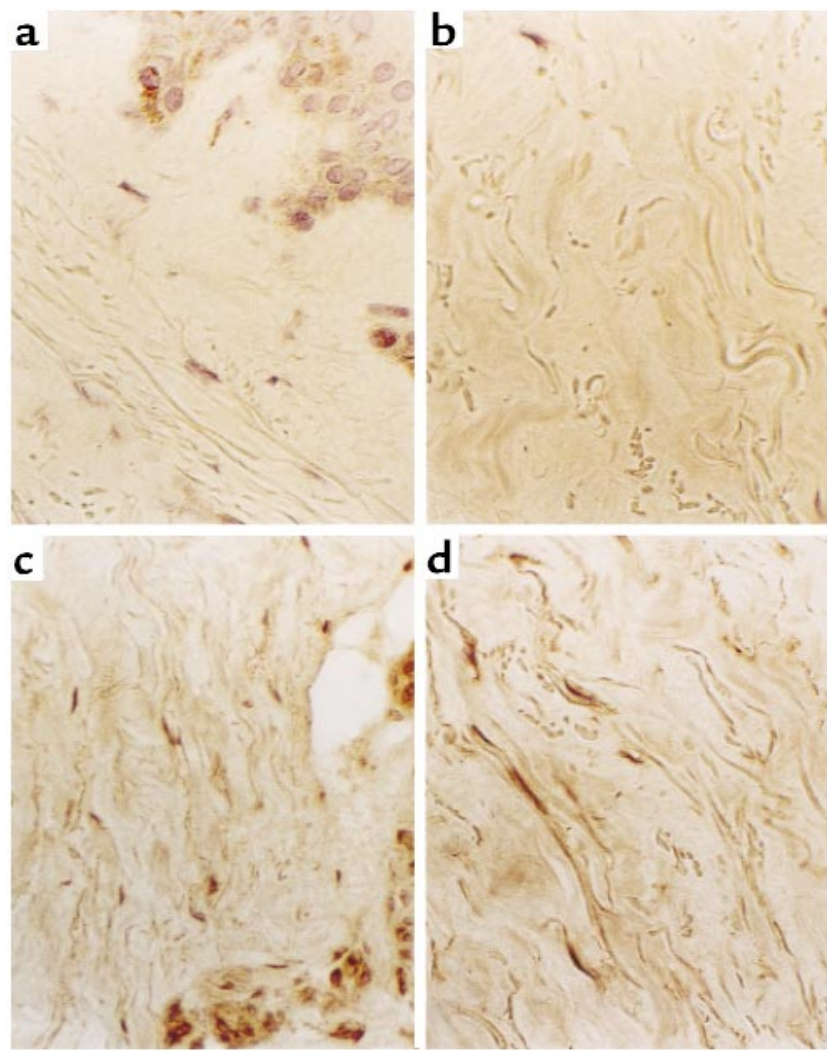

Figure 7

Immunohistochemical study for IL-1 $\alpha$ in SSc skin. Frozen sections of skin biopsies from healthy controls ( $\mathbf{a}$ and $\mathbf{b}$ ) and patients with SSc ( $\mathbf{c}$ and $\mathbf{d}$ ) were stained using anti-human IL- $1 \alpha$ antibody as described in Methods. Sections of SSc skin with preimmune rabbit IgG as a control did not show detectable staining (data not shown).

$1 \alpha$ results in increased IL-6 and PDGF-A expression in SScaffected fibroblasts. Both IL-6 and PDGF-A are key mediators of collagen synthesis and mitogenic action of fibroblasts. The evidence suggests that increased collagen synthesis can be partially mediated by IL- $1 \alpha$-inducible factors. Although we did not find extracellular release of IL-1 $\alpha$ in the previous study, the action of IL- $1 \alpha$ could occur within the cells, and immunologic functions might be involved in the abnormal phenotype of SSc-affected fibroblasts.

\section{Acknowledgments}

This study was supported by grants from the National Institutes of Health (AR-44266) and the Scleroderma Research Fund; a Research Award from the Arthritis Foundation, Western Pennsylvania Chapter; and a Research Fellowship Award from the Uehara Memorial Foundation.

1. Medsger, T.A., Jr. 1993. Systemic sclerosis (scleroderma), localized forms of scleroderma, and calcinosis. In Arthritis and allied conditions. D.J. McCarty and W.J. Koopman, editors. Lea \& Febiger. Philadelphia, PA. 1253-1292.

2. Rodnan, G.P., Lipinski, E., and Luksick, J. 1979. Skin thickness and collagen content in progressive systemic sclerosis and localized scleroderma. Arthritis Rheum. 22:130-140.

3. LeRoy, E.C. 1974. Increased collagen synthesis by scleroderma skin fibroblasts in vitro: a possible defect in the regulation or activation of the scleroderma fibroblasts. J. Clin. Invest. 54:880-889.

4. Buckingham, R.B., Prince, R.K., Rodnan, G.P., and Taylor, F. 1978. Increased collagen accumulation in dermal fibroblast cultures from patients with progressive systemic sclerosis (scleroderma). J. Lab. Clin. Med. 92:5-21.
5. Kulozik, M., Hogg, A., Lankat-Buttgereit, B., and Krieg, T. 1990. Colocalization of transforming growth factor $\mathrm{b} 2$ with a1(I) procollagen mRNA in tissue sections of patients with systemic sclerosis. J. Clin. Invest. 86:917-922.

6. Trojanowska, M., Wu, L., and LeRoy, E.C. 1988. Elevated expression of c-myc proto-oncogene in scleroderma fibroblasts. Oncogene. 3:477-481.

7. Whiteside, T.L., Ferrarini, M., Hebda, P., and Buckingham, R.B. 1988. Heterogenous synthetic phenotype of cloned scleroderma fibroblasts may be due to aberrant regulation in the synthesis of connective tissue. Arthritis Rheum. 31:1221-1229.

8. Yamakage, A., Kikuchi, K., Smith, E.A., LeRoy, E.C., and Trojanowska, M. 1992. Selective upregulation of platelet-derived growth factor a receptors by transforming growth factor b in scleroderma fibroblasts. J. Exp. Med. 175:1227-1234.

9. Kawaguchi, Y., et al. 1994. Increased endothelin-1 production in fibroblasts derived from patients with systemic sclerosis. Ann. Rheum. Dis. 53:506-510.

10. Feghali, C.A., Bost, K.L., Boulware, D.W., and Levy, L.S. 1994. Control of IL-6 expression and response in fibroblasts from patients with systemic sclerosis. Autoimmunity. 17:309-318.

11. Luger, T.A., Stadler, B.M., Katz, S.I., and Oppenheim, J.J. 1981. Epidermal cell (keratinocyte) derived thymocyte activating factor. J. Immunol. 127:1493-1498.

12. Yamato, K., el-Hajjaoui, Z., and Koeffler, H.P. 1989. Regulation of level of IL-1 mRNA in fibroblasts. J. Cell. Physiol. 139:610-616.

13. Auron, P.E., et al. 1987. Studies on the molecular nature of human interleukin 1. J. Immunol. 138:1447-1456.

14. Mauviel, A., Temime, N., Charron, D., Loyau, G., and Pujol, J.-P. 1988. Interleukin-1a and $\mathrm{b}$ stimulate interleukin-1b gene expression in human dermal fibroblasts. Biochem. Biophys. Res. Commun. 156:1209-1214.

15. Elias, J.A., and Lentz, V. 1990. IL-1 and tumor necrosis factor synergistically stimulate fibroblast IL- 6 production and stabilize IL- 6 messenger RNA. J. Immunol. 145:161-166.

16. Shroder, J.-M., Sticherling, M., Henneicke, H.H., Preissner, W.C., and Christophers, E. 1990. IL-1a or tumor necrosis factor-a stimulate release of three NAP/IL-8-related neutrophil chemotactic proteins in human dermal fibroblasts. J. Immunol. 144:2223-2232.

17. Last-Barney, K., Homon, C.A., Faanes, R.B., and Merluzzi, V.J. 1988. Synergistic and overlapping activities of tumor necrosis factor-a and IL-1. J. Immunol. 141:527-530.

18. Zucali, J.R., et al. 1986. Interleukin-1 stimulates fibroblasts to produce granulocyte-macrophage colony-stimulating activity and prostaglandin E2. J. Clin. Invest. 77:1857-1863.

19. Raines, E.W., Dower, S.K., and Ross, R. 1989. Interleukin-1 mitogenic activity for fibroblasts and smooth muscle cells is due to PDGF-AA. Science. 243:393-396.

20. Kawaguchi, Y. 1994. IL-1a gene expression and protein production by fibroblasts from patients with systemic sclerosis. Clin. Exp. Immunol. 97:445-450.

21. Mosley, B., et al. 1987. The interleukin-1 receptor binds the human interleukin-1a precursor but not the interleukin-1b precursor. J. Biol. Chem. 262:2941-2944

22. Subcommittee for Scleroderma Criteria of the American Rheumatism Association Diagnostic and Therapeutic Criteria Committee. 1980. Preliminary criteria for the classification of systemic sclerosis (scleroderma). Arthritis Rheum. 23:581-590.

23. Kawaguchi, Y., et al. 1992. Effect of prolyl 4-hydroxylase inhibitor on fibroblast collagen production in vitro: an approach to the treatment of systemic sclerosis. J. Rheumatol. 19:1710-1715.

24. Maier, J.A.M., Voulalas, P., Roeder, D., and Maciag, T. 1990. Extension of the life-span of human endothelial cells by an interleukin- $1 \alpha$ antisense oligomer. Science. 249:1570-1574.

25. Wang, A.M., Doyle, M.V., and Mark, D.F. 1989. Quantitation of mRNA by the polymerase chain reaction. Proc. Natl. Acad. Sci. USA. 86:9717-9721.

26. Burch, R.M., and Mahan, L.C. 1991. Oligonucleotides antisense to the interleukin 1 receptor mRNA block the effects of interleukin 1 in cultured murine and human fibroblasts and in mice. J. Clin. Invest. 88:1190-1196.

27. van der Krol, A.R., Mol, J.N.M., and Stuitje, A.R. 1988. Modulation of eukaryotic gene expression by complementary RNA or DNA sequences. Biotechniques. 6:958-976.

28. Marcus-Sekura, C.J. 1988. Techniques for using antisense oligodeoxyribonucleotides to study gene expression. Anal. Biochem. 172:289-295.

29. Walder, R.Y., and Walder, J.A. 1988. Role of RNase H in hybrid-arrested translation by antisense oligonucleotides. Proc. Natl. Acad. Sci. USA. 85:5011-5015.

30. Boiziau, C., et al. 1991. Inhibition of translation initiation by antisense oligonucleotides via an RNase-H independent mechanism. Nucleic Acids Res. 19:1113-1119.

31. Kawaguchi, Y., et al. 1993. Interleukin 1 receptor on fibroblasts from systemic sclerosis patients induces excessive functional responses to interleukin 1b. Biochem. Biophys. Res. Commun. 190:154-161.

32. Kadono, T., Kikuchi, K., Ihn, H., Takehara, K., and Tamaki, K. 1998. 
Increased production of interleukin 6 and interleukin 8 in scleroderma fibroblasts. J. Rheumatol. 25:296-301.

33. Hasegawa, M., et al. 1998. Serum levels of IL-6, oncostatin M, soluble IL6 receptor, and soluble gp130 in patients with systemic sclerosis. J. Rheumatol. 25:308-313.

34. Gudbjornsson, B., et al. 1994. Phenotypic and functional activation of alveolar macrophages, T lymphocytes and NK cells in patients with systemic sclerosis and primary Sjogren's syndrome. Ann. Rheum. Dis. 53:574-579.

35. Burch, R.M., and Mahan, L.C. 1991. Oligonucleotides antisense to the interleukin 1 receptor mRNA block the effects of interleukin 1 in cultured murine and human fibroblasts and in mice. J. Clin. Invest. 88:1190-1196. 36. Stein, C.A., Subasinghe, C., Shinozuka, K., and Cohen, J.S. 1988. Physicochemical properties of phosphorothioate oligodeoxynucleotides. Nucleic Acids Res. 16:3209-3221.
37. Kahari, V.M., Heino, J., and Vuorio, E. 1987. Interleukin-1 increases collagen production and mRNA levels in cultured skin fibroblasts. Biochim. Biophys. Acta. 929:142-147.

38. Duncan, M.R., and Berman, B. 1991. Stimulation of collagen and glycosaminoglycan production in cultured human adult dermal fibroblasts by recombinant human interleukin 6. J. Invest. Dermatol. 97:686-692.

39. Kulozik, M., Hogg, A., Lankat-Buttgereit, B. and Krieg, T. 1990. Co-localization of transforming growth factor $\mathrm{b} 2$ with a1(I) procollagen mRNA in tissue sections of patients with systemic sclerosis. J. Clin. Invest. 86:917-922.

40. Needleman, B.W., Wigley, F.M., and Stair, R.W. 1992. Interleukin-1, interleukin-2, interleukin-4, interleukin-6, tumor necrosis factor a, and interferon-g, levels in sera from patients with scleroderma. Arthritis Rheum. 35:67-72 